\title{
The Magnetic Switch as the Origin of the FR I/FR II Break
}

\author{
D. L. Meier, P. Godon, \& S. Edgington \\ Jet Propulsion Laboratory, Caltech, Pasadena, CA 91109, U.S.A.
}

D. G. Payne

Intel Corporation, Hillsboro, OR 97124, U.S.A.

K. R. Lind

Digital Equipment Corporation, LLNL, L-560, Livermore, CA 94550, U.S.A.

\begin{abstract}
We propose that the Fanaroff \& Riley division between class I and class II radio sources has its origins in the coronae of magnetized accretion disks around black holes. The "magnetic switch", an effect that critically controls the speed of jets produced by such disks, can explain both qualitative and quantitative differences in the properties of the two classes. These include why morphology and jet speed are functions of radio power and why the division between the two classes is a strong function of galaxy optical luminosity.
\end{abstract}

As shown by Fanaroff and Riley (1974), extragalactic radio sources can be divided into two groups, customarily called FR classes I and II. FR I sources are generally of low luminosity $\left(P_{1400 M H z} \lesssim 10^{25} \mathrm{~W} \mathrm{~Hz}^{-1}\right)$ and are characterized by strong radio emission close to the host galaxy. Morphologically this class is quite diverse, including straight, S-shaped, C-shaped, and wide and narrow angle head-tail sources. On the other hand, FR II sources have high luminosity and homogeneous morphology: nearly all are straight double sources with the two peaks radio emission occurring at each end of the double. The division in radio power is especially sharp when galaxies of the same optical magnitude are considered. Furthermore, brighter, more massive galaxies undergo the FR I/FR II transition at a significantly higher radio luminosity than fainter galaxies: $\log P_{\text {radio }}^{\text {break }}=-0.66 M_{24.5}+10.35$ or $P_{\text {radio }}^{\text {break }} \propto L_{\text {opt }}^{1.65}$, where $M_{24.5}$ is an isophotal optical magnitude (Ledlow \& Owen 1996, and references therein). We call this the OLWL (Owen-Laing-White-Ledlow) relation.

The physical interpretation of these results is that both types of source are produced by twin jets ejected from an "engine" in the center of the galaxy, but the jets in FR I sources are transonic or subsonic (Mach number $\mathcal{M} \lesssim 1-2$ ) while those in FR II sources are supersonic $(\mathcal{M} \gg \infty)$, with the transition in jet speed occurring at $V_{\text {jet }} \approx 0.6 c$ or $\gamma \approx 1.25$ (Bicknell 1985, and references therein). The speed of kiloparsec scale jets, derived from observations (Wardle \& Aaron 1997) and from dynamical interpretation of head-tail sources indicate speeds of $\sim 0.1 c(\gamma \sim 1.005)$ for FR Is and $0.6-0.8 c(\gamma \approx 1.25-1.67)$ for FR IIs. Results for parsec scale jets are very limited (because these speeds are generally determined for sources pointing close to the line of sight, making determination of the kiloparsec scale morphology difficult), but indicate a possible trend with $\gamma \lesssim 3$ for FR Is and $\gamma \approx 2-10$ for FR IIs (Pearson 1996). If this is borne out with further observations, it would indicate that the origin of the FR I/FRII division lies near or in the central engine itself.

Recently the authors have undertaken a large numerical study of one of the most promising models for producing jets in radio sources and other objects-... the Blandford \& Payne (1982) magnetohydrodynamic acceleration mechanism in rotating accretion disks around black holes. Centrifugal action in accretion disks, threaded with a poloidal magnetic field, flings matter up along the field lines, 
wraps the field around the plasma, and collimates the outflow into a slender jet. The most important results from our work so far are: 1$)$ in the corona above the accretion disk, where the density $\rho$ is low, the Alfvén velocity $\left(V_{A} \equiv B /(4 \pi \rho)^{1 / 2}\right)$ can be quite high, even for modest disk magnetic field strengths $B$; and 2 ) there is a sharp difference in the speed of the jet produced for disk coronae with $V_{A}<V_{e s c} \equiv\left(2 G M_{B H} / R_{0}\right)^{1 / 2}$ and those with $V_{A}>V_{e s c}$, where $M_{B H}$ is the mass of the black hole and $R_{0}$ is the radius in the disk where $B$ has its maximum value $B_{0}$ (Meier et al. 1997). Disks with $\nu \equiv V_{A} / V_{e s c}<1$ produce jet velocities of only a few tenths $c$, but disks with $\nu>1$ produce very high velocity jets $(\gamma \gtrsim 10)$. However, there is no such strong transition in jet power at $\nu=1$, which varies smoothly across the switch as $P_{j e t} \approx B_{0}^{2} R_{0}^{2} V_{\text {esc }}$.

We propose that this effect, called the "magnetic switch", is the origin of the FRI/FR II division. It explains why a strong change in jet speed occurs at a given radio power: as the magnetic field in the disk corona is increased, both the $V_{A}$ (and therefore $V_{j e t}$ ) and the jet power $P_{\text {jet }}$ increase, but only $V_{\text {jet }}$ is a very strong function of magnetic field strength. Furthermore, it can quantitatively explain the slope of the OLWL relation. Using the expression for jet power above and our theoretical expression for the FR I/FR II break $(\nu=1)$, and assuming that coronal density scales with disk density, we obtain a theoretical expression for jet power at the break of $P_{j e t}^{b r e a k} \propto M_{B H}^{1.3}$, with the -0.7 in the exponent of $M_{B H}$ coming from Shakura \& Sunyaev's (1973) accretion disk models. To relate black hole mass to galaxy optical luminosity, we use the well-known Kormendy \& Richstone (1995) relation $\left(M_{B H} \propto L_{\text {opt }}^{1.25}\right)$, yielding a predicted trend of $P_{j e t}^{b r e a k} \propto L_{o p t}^{1.625}$, in very close agreement with the slope of the OLWL relation. Without better models of the disk corona, it is difficult to theoretically predict the intercept of the OLWL relation. However, we find that the average observed break power of $\sim 10^{25} \mathrm{WH}^{-1}$ is consistent with $\nu=1$ in a plasma with density many orders of magnitude smaller than standard accretion disk models, in agreement with the original model assumption that jets are formed in low density coronae rather than in the disks themselves.

Acknowledgments. Part of this research was carried out at the Jet Propulsion Laboratory, California Institute of Technology, under contract to NASA. S. E. Acknowledges the support of an Associated Western Universities Summer Fellowship. During this research, P. G. held a National Research Council Research Associateship at NASA JPL.

\section{References}

Bicknell, G. V. 1985. Proc. Astr. Soc. Austr., 6, 130-137.

Blandford, R. D., \& Payne, D. G. 1982. MNRAS, 199, 883-903.

Fanaroff, B. L., \& Riley, J. M. 1974. MNRAS, 167, 31P-35P.

Meier, D. L., et al. 1997. Nature, 388, 350 352.

Kormendy, J., \& Richstone, D. 1995. ARA\&A, 33, 581-624.

Ledlow, M. J., \& Owen, F. N. 1996. AJ, 112, 9-22.

Pearson, T. J. 1996. In Energy Transport in Radio Galaries and Quasars, eds. P. E. Hardee, A. H. Bridle, \& J. A. Zensus (San Fransico: ASP), 97-108.

Shakura, N. I., \& Sunyaev, R. A. 1973. $A \& A, 24,337-355$.

Wardle, J. F. C., \& Aaron, S. E. 1997. MNRAS, 286, 425-435. 